\title{
Diagnostic and Prognostic Value of Neutrophil Extracellular Trap Levels in Patients With Acute Aortic Dissection
}

\author{
Shuofei Yang ${ }^{1 \dagger}$, Yongsheng Xiao ${ }^{2 \dagger}$, Yuanfeng $\mathrm{Du}^{3}$, Jiaquan Chen ${ }^{1}$, Qihong $\mathrm{Ni}^{1}$, \\ Xiangjiang Guo ${ }^{1}$, Guanhua Xue ${ }^{1 *}$ and Xupin Xie ${ }^{4 *}$ \\ ${ }^{1}$ Department of Vascular Surgery, Renji Hospital, School of Medicine, Shanghai Jiaotong University, Shanghai, China, \\ ${ }^{2}$ Department of Vascular Surgery, Tianjin 4th Centre Hospital, The Fourth Central Hospital Affiliated to Nankai University, The \\ Fourth Center Clinical College of Tianjin Medical University, Tianjin, China, ${ }^{3}$ Department of Neurosurgery, School of Medicine, \\ Affiliated Hangzhou First People's Hospital, Zhejiang University, Hangzhou, China, ${ }^{4}$ Department of Vascular Surgery, School \\ of Medicine, Affiliated Hangzhou First People's Hospital, Zhejiang University, Hangzhou, China
}

OPEN ACCESS

Edited by:

Alessio Molfino,

Sapienza University of Rome, Italy

Reviewed by:

Ruijing Zhang,

Second Hospital of Shanxi Medical

University, China

Kumaravelu Jagavelu,

Central Drug Research Institute

(CSIR), India

*Correspondence:

Guanhua Xue

xueguanhua2018@163.com

Xupin Xie

xiexupin@163.com

tThese authors have contributed equally to this work and share first authorship

Specialty section:

This article was submitted to General Cardiovascular Medicine,

a section of the journal

Frontiers in Cardiovascular Medicine

Received: 21 March 2021 Accepted: 10 September 2021 Published: 15 February 2022

Citation:

Yang S, Xiao Y, Du Y, Chen J, Ni Q,

Guo X, Xue G and Xie X (2022)

Diagnostic and Prognostic Value of

Neutrophil Extracellular Trap Levels in

Patients With Acute Aortic Dissection.

Front. Cardiovasc. Med. 8:683445.

doi: 10.3389/fcvm.2021.683445
Background: Acute aortic dissection (AAD) is a fatal disease demanding prompt diagnosis and proper treatment. There is a lack of serum markers that can effectively assist diagnosis and predict prognosis of AAD patients.

Methods: Ninety-six AAD patients were enrolled in this study, and 249 patients with chest pain due to acute myocardial infarction, pulmonary embolism, intramural hematoma, angina or other causes and 80 healthy controls were included as control group and healthy control group. Demographics, biochemical and hematological data and risk factors were recorded as baseline characteristics. The 1-year follow-up data were collected and analyzed. The diagnostic performance and ability to predict disease severity and prognosis of NET components in serum and aortic tissue were evaluated.

Results: Circulating NET markers, citH3 (citrullination of histone 3), cell-free DNA (cfDNA) and nucleosomes, had good diagnostic value for AAD, with superior diagnostic performance to D-dimer in discriminating patients with chest pain due to other reasons in the emergency department. Circulating NET marker levels (i.e., citH3, cfDNA and nucleosomes) of AAD patients were significantly higher than that of control group and healthy control group. In addition, circulating NET markers levels were closely associated with the disease severity, in-hospital death and 1-year survival of AAD patients. Systolic blood pressure $<90 \mathrm{mmHg}$ and serum citH3 levels were identified as independent risk factors for 1-year survival of AAD patients. Excessive NET components (i.e., neutrophil elastase and citH3) in the aortic tissue of AAD patient were significantly higher than that of healthy donor aortic tissue. The expression levels of granules and nuclear NET components were significantly higher in aortic tissue from AAD patients than controls.

Conclusions: Circulating NET markers, citH3, cfDNA and nucleosomes, have significant diagnostic value and predictive value of disease severity and prognosis of AAD patients. The NETs components may constitute a useful diagnostic and prognostic marker in $A A D$ patients.

Keywords: neutrophil extracellular trap, acute aortic dissection, serum biomarker, diagnostic marker, prognostic marker 


\section{INTRODUCTION}

Acute aortic dissection (AAD) is a fatal aortic disease with high mortality and morbidity that demands prompt diagnosis and proper treatment (1). Despite recent advances in diagnostic imaging methods, AAD remains a challenge to diagnose. A widely available and cost-effective measure such as a blood test that can rule in and/or rule out the disease would indeed aid in quick diagnosis, benefiting patients and caregivers. Several major diseases that cause chest pain, such as acute myocardial infarction (AMI), pulmonary embolism (PE), intramural hematoma and angina, require differential diagnosis with $\mathrm{AAD}$. Moreover, there is still a lack of effective markers to accurately predict the inhospital and long-term outcomes of patients with AAD after surgical repair.

Neutrophils are the most abundant cell type in leukocytes and play a crucial role in the innate immune system (2). Neutrophils are also involved in the pathological mechanism of aortic dissection (AD) (3). AAD is initiated by neutrophil infiltration of the aortic intima, and local neutrophil recruitment and activation in response to AAD can lead to aortic rupture (4, 5). In patients with $A A D$ receiving surgical repair, the neutrophil to lymphocyte ratio may be used to predict worse outcomes and hospital mortality (6). In the inflammatory response, neutrophils play critical roles through the release of neutrophil extracellular traps (NETs), which are extracellular neutrophil-derived weblike structures that constitute a DNA backbone containing histones and neutrophil granule proteins. A critical step in NET release is the citrullination of histone 3 ( $\mathrm{citH} 3$ ), a process mediated by protein deiminase 4 , and citH3 is considered one of the most specific markers for NET formation assessment (7). Initially, NETs were thought to provide defense against pathogens (8). In recent years, NETs have been implicated in a number of cardiovascular diseases (9). By immunophenotypic analysis, NETs were found to participate in the tissue repair of $\mathrm{AD}(10)$.

NETs could be used as a new circulating marker for several cardiovascular diseases, such as acute coronary syndrome, acute ischemic stroke, myocardial infarction, and deep venous thrombosis (11-13). Data on NET presence in the serum and tissue of AAD patients and the association between NET levels and clinical outcomes are scarce. Hence, this study sought to determine whether NETs may serve as disease biomarkers in AAD patients. Specifically, our aims were to examine the diagnostic value of NETs for AAD and the predictive significance of NETs for disease severity, in-hospital mortality and 1-year survival in AAD patients receiving surgical repair.

Abbreviations: AAD, acute aortic dissection; AMI, acute myocardial infarction; $\mathrm{AD}$, aortic dissection; PE, pulmonary embolism; NETs, neutrophil extracellular traps; citH3, citrullination of histone 3; CTA, computed tomography angiography; MRA, magnetic resonance angiography; TAAD, type A aortic dissection; TABD, type B aortic dissection; APACHE II, the Acute Physiology, Age, Chronic Health Evaluation II; cfDNA, cell-free DNA; NE, neutrophil elastase; ROC, receiver operating characteristic curve; AUR, area under the ROC; NET, neutrophil extracellular trap; SBP, systolic blood pressure; TF, tissue factor.

\section{PATIENTS AND METHODS}

\section{Patients}

The study was approved by the ethical committee of Renji Hospital, School of Medicine, Shanghai Jiaotong University (No. RA2020-253). All patients or their proxies provided written informed consent. Ninety-six consecutive patients with AAD hospitalized in Renji Hospital between May 01, 2016, and April 04, 2019, were enrolled in this study. Diagnoses were made based on computed tomography angiography (CTA), digital subtraction angiography and, when appropriate, magnetic resonance angiography (MRA). The Stanford classifications of AAD were evaluated at the time of diagnosis. Stanford type A aortic dissection (TAAD) involves the ascending aorta, whereas type $\mathrm{B}$ aortic dissection (TABD) involves the descending aorta only. The severity of disease was measured by the Acute Physiology, Age, Chronic Health Evaluation II (APACHE II) score at hospital entrance and discharge. The detection risk score of $\mathrm{AD}$ was calculated at admission to the emergency department according to the guidelines (14). During the same time period, 249 patients admitted to the emergency chest pain center with a diagnosis of AMI, PE, angina or others were included in the control group. In addition, 80 healthy controls were included in this study. Demographics, biochemical and hematological data, clinical history, and risk factors were recorded as baseline patient characteristics. Clinical follow-up was performed 1 year after hospital discharge in all AAD patients. This was conducted by reviewing the electronic records in clinics or by telephone contact. Follow-up records included reoperation information, CTA or MRA follow-up information, mortality, and cause and date of death.

Citrated-anticoagulated venous blood was obtained from all the patients within $3 \mathrm{~h}$ of diagnosis and on the last morning before discharge. Platelet-poor plasma was prepared by centrifugation of the blood $(2,500 \times \mathrm{g})$ for $10 \mathrm{~min}$ at $22^{\circ} \mathrm{C}$, and the plasma was stored at $-80^{\circ} \mathrm{C}$ until analysis. Aortic tissue samples were obtained from $45 \mathrm{AAD}$ patients included in this study who received open surgery. Twenty normal aortic tissues were obtained from organ donors (crash victims or brain-dead patients). Tissue samples were stored at $-80^{\circ} \mathrm{C}$ until analysis.

\section{NET Markers}

We evaluated three different markers of NETs [i.e., cfDNA (cell-free DNA), nucleosomes and citH3]. citH3 is currently considered to be the most specific marker, as $\mathrm{H} 3$ citrullination is required for chromatin decondensation in neutrophils. Detection of cfDNA and nucleosomes was performed as described previously (13). For cfDNA determination, plasma was diluted 1:10 with phosphate-buffered saline and mixed with an equal volume of $1 \mathrm{mM}$ SytoxGreen (Invitrogen, Carlsbad, CA, USA; No. S7020) in PBS. Fluorescence was determined in a fluorescence microplate reader (Gemini XPS; Molecular Devices, Sunnyvale, CA, USA). A calibration curve was generated with calf thymus DNA (Invitrogen; No. 15633019) in PBS. Nucleosomes were measured with the Cell Death Detection ELISAPLUS kit (Roche Diagnostics, Madrid, Spain; No. 11774425001) according to the manufacturer's instructions. Determination of citH3 was 
TABLE 1 | Baseline characteristics of chest pain patients included in this study.

\begin{tabular}{|c|c|c|c|}
\hline Characteristics & $\begin{array}{c}\text { AAD } \\
(n=96)\end{array}$ & $\begin{array}{l}\text { Controls } \\
(n=249)\end{array}$ & $P$-value \\
\hline Age, mean (range) & $59.1(35-85)$ & $60.3(40-92)$ & 0.45 \\
\hline Male sex, $n(\%)$ & $53(55.2)$ & $128(51.4)$ & 0.53 \\
\hline \multicolumn{4}{|c|}{ Medical history and risk factors, $n$ (\%) } \\
\hline Hypertension & $72(75.0)$ & $129(51.8)$ & $<0.05$ \\
\hline Diabetes & $44(45.8)$ & $109(43.8)$ & 0.62 \\
\hline Stroke & $28(29.2)$ & $80(32.1)$ & 0.60 \\
\hline Hyperlipidemia & $48(50.0)$ & $135(54.2)$ & 0.48 \\
\hline Smoking & $55(57.3)$ & $125(50.2)$ & 0.24 \\
\hline Marfan syndrome & $12(12.5)$ & $1(0.4)$ & $<0.01$ \\
\hline Atrial fibrillation & $28(29.2)$ & $63(25.3)$ & 0.47 \\
\hline Valvulopathy & $20(20.8)$ & $42(16.9)$ & 0.39 \\
\hline \multicolumn{4}{|l|}{ Diagnosis, $n$ (\%) } \\
\hline TAAD & $42(43.8)$ & / & / \\
\hline TBAD & $54(56.3)$ & / & / \\
\hline $\mathrm{IH}$ & / & $10(4.0)$ & / \\
\hline AMl & / & $63(25.3)$ & / \\
\hline PE & / & $42(16.9)$ & / \\
\hline Angina & / & $66(26.5)$ & / \\
\hline Others & / & $68(27.3)$ & / \\
\hline \multicolumn{4}{|c|}{ Biochemical and hematological data, mean $\pm S D$} \\
\hline Glucose, mg/dL & $130.3 \pm 52.4$ & $125.4 \pm 44.2$ & 0.42 \\
\hline Creatinine, $\mu \mathrm{mol} / \mathrm{L}$ & $148.7 \pm 33.6$ & $142.9 \pm 40.7$ & 0.36 \\
\hline Uric acid, mg/dL & $6.1 \pm 2.4$ & $5.9 \pm 2.9$ & 0.37 \\
\hline Cholesterol, mg/dL & $178.3 \pm 39.2$ & $183.2 \pm 29.9$ & 0.72 \\
\hline Triglycerides, mg/dL & $182.1 \pm 88.4$ & $189.1 \pm 79.2$ & 0.60 \\
\hline AST, U/L & $28.2 \pm 13.4$ & $30.2 \pm 20.2$ & 0.13 \\
\hline $\mathrm{ALT}, \mathrm{U} / \mathrm{L}$ & $38.3 \pm 16.7$ & $39.6 \pm 26.3$ & 0.22 \\
\hline Bilirubin, mg/dL & $1.9 \pm 1.6$ & $1.8 \pm 0.8$ & 0.82 \\
\hline Hematocrit, \% & $41.3 \pm 6.3$ & $39.4 \pm 5.6$ & 0.63 \\
\hline Platelets, $10^{3} / \mu \mathrm{L}$ & $248.3 \pm 56.4$ & $256.6 \pm 47.2$ & 0.20 \\
\hline Leukocytes, $10^{3} / \mu \mathrm{L}$ & $6.4 \pm 3.4$ & $6.8 \pm 3.9$ & 0.74 \\
\hline Lymphocytes, \% & $26.3 \pm 9.2$ & $27.5 \pm 8.4$ & 0.20 \\
\hline Neutrophils, \% & $74.4 \pm 18.3$ & $73.9 \pm 19.8$ & 0.48 \\
\hline Monocytes, \% & $8.8 \pm 4.2$ & $9.0 \pm 7.3$ & 0.33 \\
\hline D-dimer, ng/ml & $2080.5 \pm 1131.9$ & $1867.3 \pm 2007.3$ & $<0.05$ \\
\hline
\end{tabular}

$A A D$, acute aortic dissection; TAAD, type $A$ aortic dissection; TBAD, type $B$ aortic dissection; $I H$, intramural hematoma; $A M I$, acute myocardial infarction; $P E$, pulmonary embolism; SD, standard deviation; AST, aspartate transaminase; ALT, alanine aminotransferase.

performed as previously described (15). Briefly, plasma samples were mixed with a monoclonal mouse anti-histone biotinylated antibody in a streptavidin-coated plate. A rabbit polyclonal antihistone-H3 (citrullinated R17 + R2 + R8) (Abcam Inc., MA, USA; No. ab81797) antibody was used in the second step. Detection was performed with a peroxidase-linked antibody (GE Biosciences, Barcelona, Spain; No. A1783). Values were normalized to a pool of samples from normal subjects. Values are expressed as individual absorption values. The neutrophil elastase (NE) concentration was measured using commercially available ELISA kits.

\section{Proteomics Analysis}

In this study, we built a custom pathway of NET-associated proteins as described previously (16). To build a custom "NETosis" pathway, twenty-three proteins that belonged to five subcellular compartments (nucleus, granules, cytoplasm/cytoskeleton, enzymes, and plasma membrane) were identified by a literature screen for detailed characterization of NET proteins $(17,18)$. According to a Gene Set Enrichment Analysis heat map, the custom NETosis pathway was enriched in aortic tissue from AAD patients vs. normal controls. Samples were reduced, alkylated and trypsin-digested according to the iTRAQ manufacturer's instructions (AB Sciex Inc., MA, USA). To diminish any potential variation introduced by the labeling reaction, samples from $\mathrm{AAD}$ patients and normal controls were split into two aliquots of $60 \mu \mathrm{g}$ to perform two technical replicates with tag swapping. Each peptide solution was labeled at room temperature for $1 \mathrm{~h}$ with one iTRAQ reagent vial. To verify the labeling efficiency, $1 \mu \mathrm{g}$ of each labeled sample was individually analyzed by liquid chromatography-tandem mass spectrometry (LC-MS/MS) as specified below. Acquired data were searched against the Mascot database, setting iTRAQ labeling as the variable modification. No unmodified peptides were identified from the search, and all the peptides were correctly modified at the N-terminus and at each lysine residue. Finally, the four iTRAQ-labeled samples were combined in a 1:1:1:1 ratio, and the pool was vacuum dried in a SpeedVac system.

\section{Immunofluorescence}

NET identification in tissue samples was performed by immunofluorescence staining. The NE/citH3 pair was researched in paraffin-embedded, $3-\mu \mathrm{m}$-thick sections. The slides were incubated with the primary antibodies (anti-NE antibody, MAB91671, R\&D Systems, Minneapolis, USA; anti-H3Cit antibody, ab5103, Abcam, Cambridge, UK; both 1:50 dilution) at $4^{\circ} \mathrm{C}$ overnight after blocking with goat serum. Then, the sections were incubated with secondary antibodies (Alexa Fluor 488, green, ab150077; Alexa Fluor 647, red, ab150075; both from Abcam, Cambridge, UK) for $1 \mathrm{~h}$ at room temperature. DAPI was used for nuclear staining (ZSGB Biotech, Beijing, China; No. ZLI-9577). The slides were analyzed with a confocal laser scanning microscope (TCS-SP5; Leica, Wetzlar, Germany). The average numbers of $\mathrm{NE}$ and $\mathrm{H} 3 \mathrm{Cit}$ double-positive cells were calculated by two independent researchers counting five random microscopic fields.

\section{Statistics}

Data are expressed as the means \pm SEM of absolute values or as percentages. Continuous variables were analyzed with the Mann-Whitney test. Discrete variables were evaluated with a contingency $\chi^{2}$ test. By the Shapiro-Wilk test, the value of citH3, cfDNA and nucleosomes were found to be normally distributed. The Spearman coefficient $(r)$ was used to quantify the correlations between variables. Compared with D-dimer, the diagnostic performance of NET markers for distinguishing AAD from all other diseases, AMI, PE, or angina was assessed using receiver operating characteristic curve (ROC) analysis. The area under the ROC (AUR), 
TABLE 2 | NETs and risk factors before the onset of the acute event.

\begin{tabular}{|c|c|c|c|c|c|c|c|}
\hline Characteristics & & citH3 (ng/ml) & $P$ & cfDNA (AU) & $P$ & Nucleosomes (AU) & $P$ \\
\hline \multirow[t]{2}{*}{ Hypertension } & Yes & $0.46 \pm 0.23$ & 0.73 & $698.82 \pm 306.28$ & 0.11 & $1.56 \pm 0.82$ & 0.82 \\
\hline & No & $0.47 \pm 0.26$ & & $788.13 \pm 438.75$ & & $1.58 \pm 0.88$ & \\
\hline \multirow[t]{2}{*}{ Diabetes } & Yes & $0.46 \pm 0.23$ & 0.56 & $692.48 \pm 317.45$ & 0.31 & $1.53 \pm 0.85$ & 0.75 \\
\hline & No & $0.47 \pm 0.25$ & & $745.40 \pm 365.98$ & & $1.59 \pm 0.83$ & \\
\hline \multirow[t]{2}{*}{ Smoking } & Yes & $0.48 \pm 0.24$ & 0.12 & $749.98 \pm 355.40$ & 0.21 & $1.61 \pm 0.85$ & 0.32 \\
\hline & No & $0.44 \pm 0.24$ & & $682.46 \pm 328.01$ & & $1.50 \pm 0.82$ & \\
\hline \multirow[t]{2}{*}{ Marfan syndrome } & Yes & $0.47 \pm 0.21$ & 0.52 & $664.25 \pm 210.41$ & 0.04 & $1.52 \pm 0.74$ & 0.21 \\
\hline & No & $0.47 \pm 0.24$ & & $729.27 \pm 359.05$ & & $1.57 \pm 0.85$ & \\
\hline \multirow[t]{2}{*}{ Atrial fibrillation } & Yes & $0.49 \pm 0.25$ & 0.44 & $757.68 \pm 359.99$ & 0.09 & $1.57 \pm 0.80$ & 0.71 \\
\hline & No & $0.45 \pm 0.24$ & & $706.10 \pm 338.55$ & & $1.56 \pm 0.85$ & \\
\hline
\end{tabular}

citH3, citrullination of histone 3; cfDNA, cell-free DNA; $A U$, arbitrary units.

sensitivity, specificity, accuracy, positive predictive value, and negative predictive value were calculated. The Wald test was used to assess the significance of the difference between areas under the ROC curve. The optimal cutoff point from the study was the threshold leading to the maximum summation of sensitivity and specificity. Univariate logistic regression analysis was used to assess the association between risk factors and in-hospital death or 1-year survival, and a multivariate Cox regression analysis was performed using variables with $P \leq 0.20$ in univariate analysis. A two-sided $P$ value $<0.05$ was considered statistically significant. Statistical analyses were performed with SPSS 20 (SPSS, Inc., Chicago, IL, USA).

\section{RESULTS}

\section{Baseline Demographic Information and Hematological Parameters}

A total of 96 patients, 42 TAADs and 54 TBADs, were enrolled in the AAD group. Another 249 patients were enrolled in the control group, including $10 \mathrm{IH}$ cases, 63 AMI cases, 42 PE cases, 66 angina cases and 68 cases with other causes. The baseline demographic information, medical history, risk factors, and biochemical and hematological data are shown in Table 1. The demographic information, biochemical and hematological data of 80 healthy controls are shown in Supplementary Table 1. The rates of hypertension and Marfan syndrome were significantly higher in the AAD group than in the control group. Moreover, AAD patients had significantly higher levels of $\mathrm{D}$-dimer than patients in the control group. There was no significant difference between the two groups regarding other parameters. The AAD patients were stratified according to classical risk factors. No significant differences among any of the three NET markers were observed between patients with or without hypertension, diabetes, smoking history and atrial fibrillation. The cfDNA levels in patients with Marfan syndrome were significantly higher than those in patients without Marfan syndrome. However, the levels of citH3 and nucleosomes were comparable between patients with or without Marfan syndrome (Table 2).

\section{Diagnostic Performance of Circulating NET Markers for Discriminating AAD}

The values of citH3, cfDNA and nucleosomes in patients with AAD were significantly higher than those in the control group or healthy controls (Figure 1A) Circulating levels of citH3, cfDNA, and nucleosomes were positively correlated with the detection risk score of $\mathrm{AD}$ (Figure 1B). In the ROC curve, the AURs in patients with AAD vs. all control patients were 0.87 for citH3, 0.95 for cfDNA, 0.92 for nucleosomes, and 0.64 for D-dimer (Figure 1C). Thus, circulating NET markers showed superior overall diagnostic performance compared with D-dimer when sudden-onset chest pain was present in the emergency department. cfDNA at cutoff levels of $403.5 \mathrm{ng} / \mathrm{ml}$ and D-dimer at $2015 \mathrm{ng} / \mathrm{ml}$ were the thresholds leading to the maximum summation of sensitivity and specificity in discriminating AAD from all other diagnoses. The corresponding sensitivities were $77.08 \%$ for cfDNA and $57.29 \%$ for D-dimer, and the specificities were $100 \%$ for cfDNA and $84.74 \%$ for D-dimer, resulting in 93.62\% of patients for cfDNA and $55.61 \%$ of patients for D-dimer being correctly classified (Supplementary Table 2).

The time course was also examined in the AAD group using box plot analysis according to the admission time from symptom onset. The peak NET marker levels occurred within $12-24 \mathrm{~h}$ after symptom onset (Figure 1D). In addition, no significant difference in circulating NET markers was found between patients with TAAD and TBAD or among different subsets of the control group. There was no correlation between age and circulating levels of NET markers (Supplementary Figure 1).

\section{Association Between Circulating NET Markers and Disease Severity of AAD at Onset and Discharge}

There was a positive correlation between the APACHE II score and the levels of all three NET markers at disease onset (Figure 2A). When patients were classified according to the APACHE II score into four groups, those with higher scores had significantly higher levels of all three NET markers (Figure 2B). At discharge, the levels of all three NET markers were also positively correlated with the APACHE II score (Figure 2C). Classified by APACHE II score into three groups, patients with 


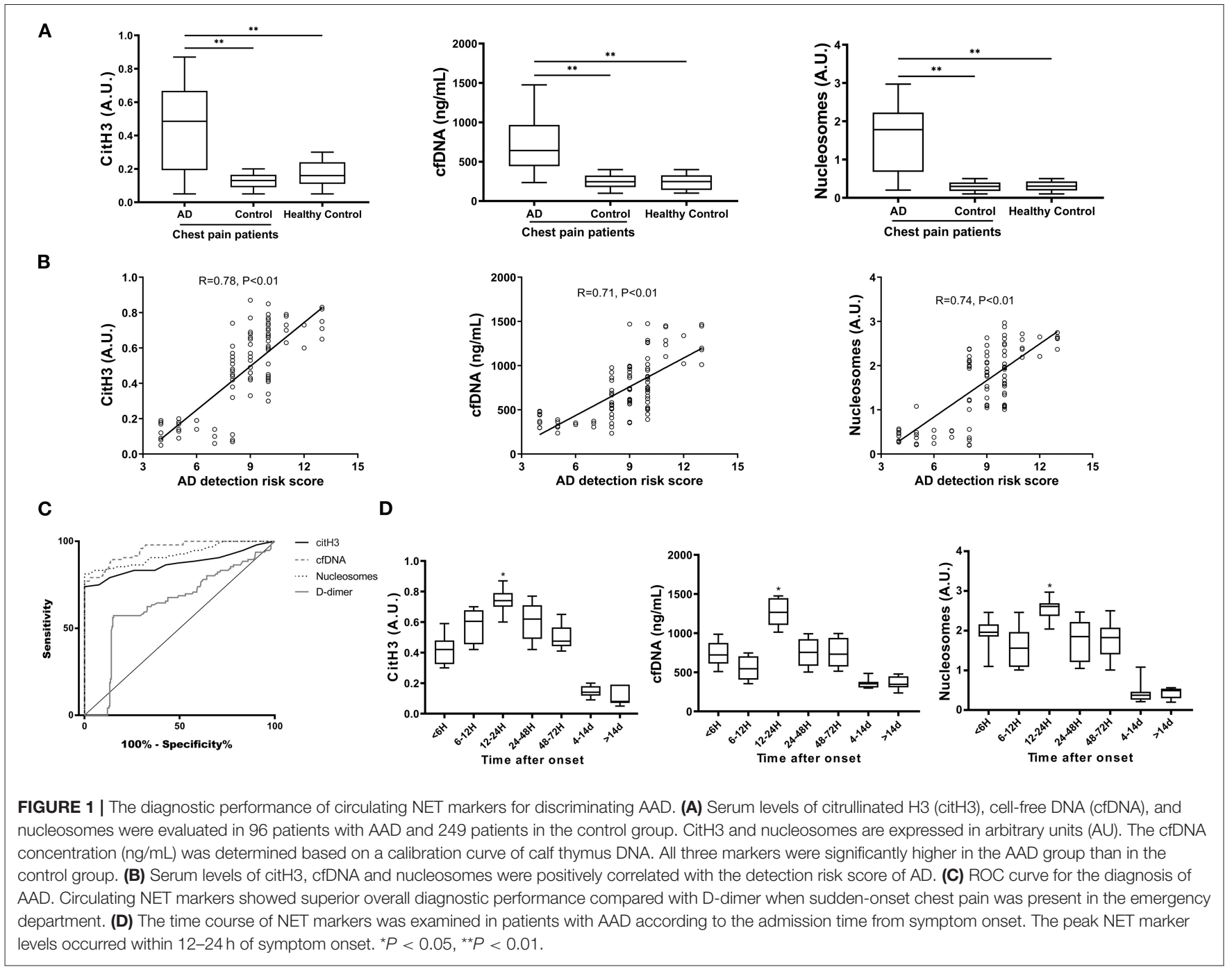

greater disease severity showed significantly higher levels of the three NET markers (Figure 2D). These results demonstrated the association between circulating levels of NET markers and disease severity at both admission and discharge.

\section{Prognostic Significance of Circulating NET Markers for Predicting In-hospital Death and 1-year Survival of Patients With AAD}

The results in Figure 3A show that the levels of NET markers in patients with in-hospital death were significantly higher than those in patients without in-hospital death. AAD patients with the highest quartiles of citH3, cfDNA, or nucleosome levels presented significantly lower survival rates by 1 year than patients with the lower three quartiles of citH3 (Figure 3B). Based on the ROC curve, three circulating NET markers showed superior predictive ability for 1-year survival compared with D-dimer (Figure 3C). The AURs were 0.72 for citH3, 0.76 for cfDNA, 0.73 for nucleosomes, and 0.51 for D-dimer. The cfDNA at cutoff levels of $1,052 \mathrm{ng} / \mathrm{ml}$ and D-dimer at $915 \mathrm{ng} / \mathrm{ml}$ were threshold values. The corresponding sensitivities were $46.15 \%$ for cfDNA and $26.92 \%$ for D-dimer, and the specificities were $94.29 \%$ for cfDNA and $82.86 \%$ for D-dimer (Supplementary Table 3).

\section{Risk Factor Analysis of 1-year Survival in AAD Patients}

We next examined whether NET markers were independently associated with the 1-year survival of AAD patients. By univariate and multivariate risk factor analysis, systolic blood pressure $(\mathrm{SBP})<90 \mathrm{mmHg}$ and citH3 levels were identified as independent risk factors for 1-year survival $(p<0.05)$ (Figure 4A and Supplementary Table 4). AAD patients with SBP $<90 \mathrm{mmHg}$ had significantly increased circulating NET markers compared with patients with SBP $>90 \mathrm{mmHg}$ (Figure 4B).

\section{Excess NET Components in Aortic Tissue and Their Association With Disease Severity and Prognosis in Patients With AAD}

The expression levels of granule and nuclear NET components were significantly higher, and non-granular enzymes and 

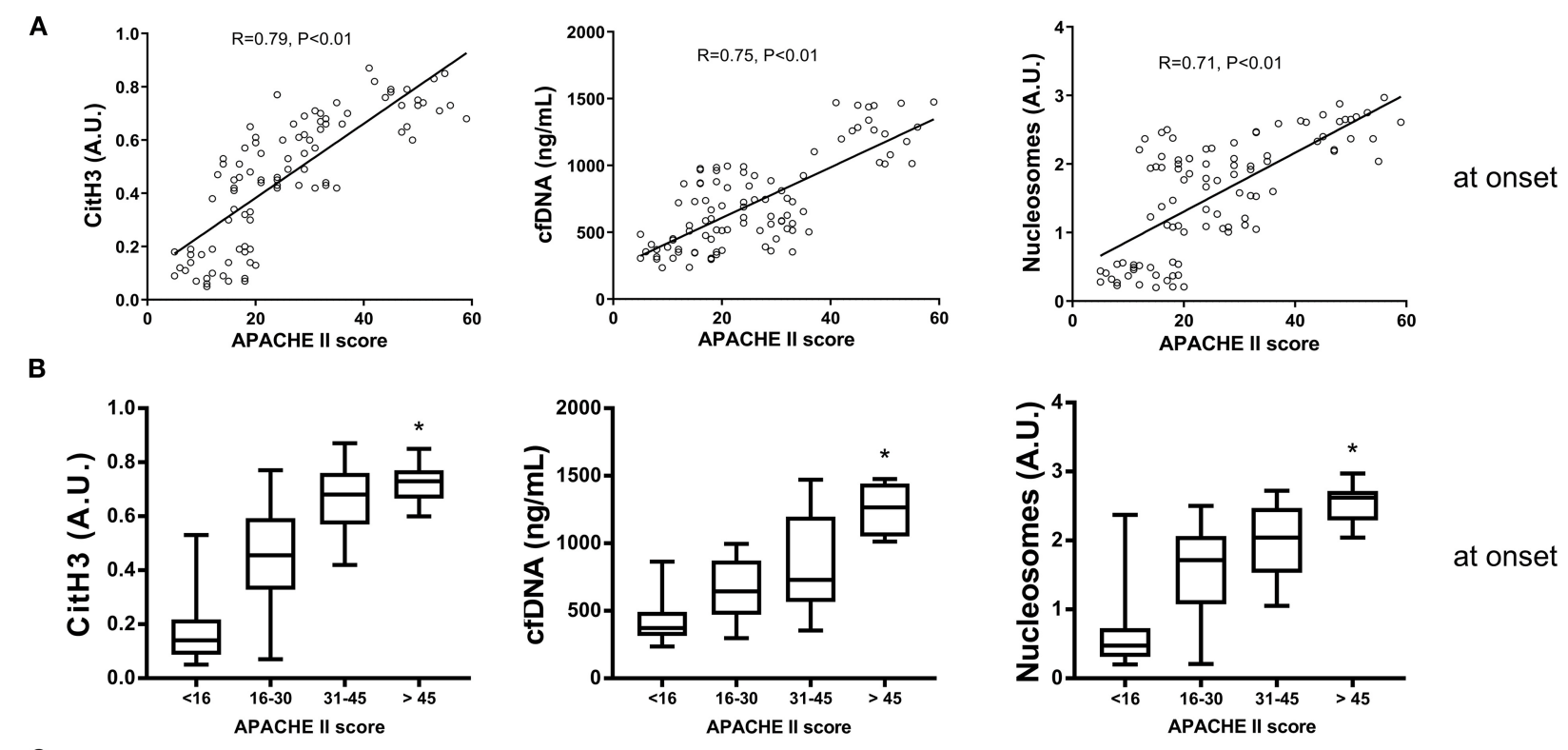

at onset

C
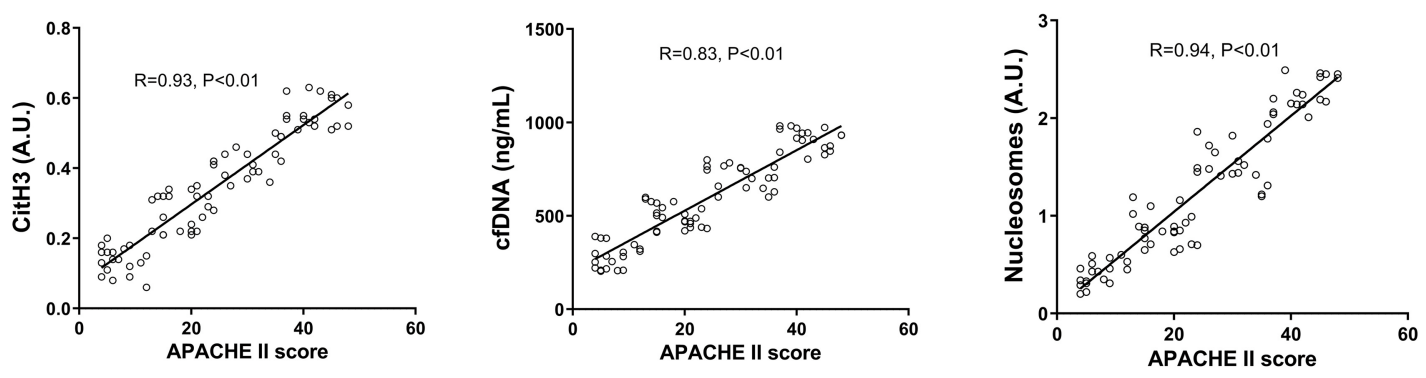

at discharge

D
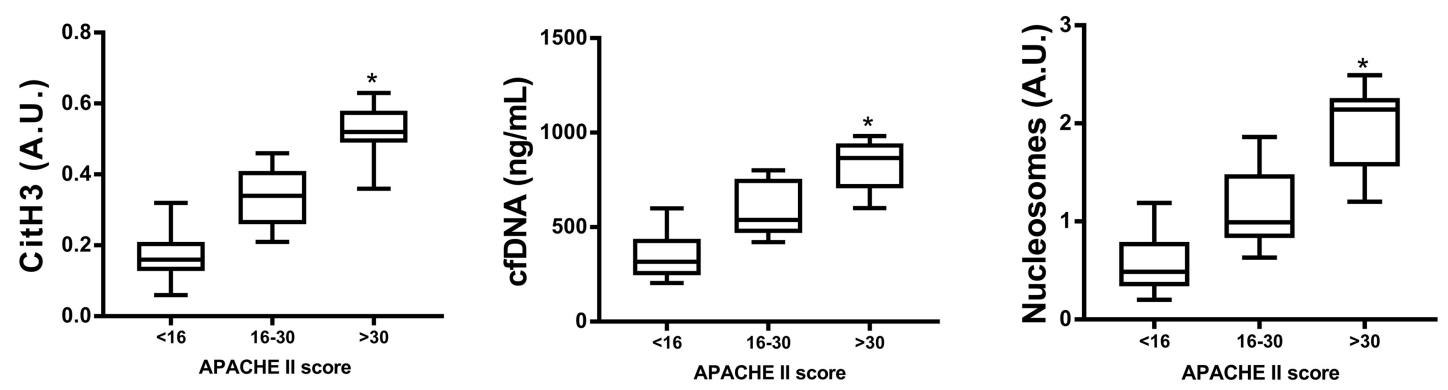

at discharge

FIGURE 2 | The association between NET markers and disease severity of AAD at onset and discharge. (A) Serum levels of citH3, cfDNA and nucleosomes were positively correlated with the APACHE II score at disease onset. (B) AAD patients were classified into four groups according to APACHE II score. Patients with higher scores had significantly higher levels of all three NET markers. (C) The levels of all three NET markers were positively correlated with the APACHE II score at discharge. (D) AAD patients with greater disease severity showed significantly higher levels of NET markers. ${ }^{*} P<0.05$.

membrane components were mildly enriched in aortic tissue from AAD patients compared with normal controls (Figures 5A,B). The expression of neutrophil elastase, the prototypical NET marker, was significantly higher in aortic tissue from the $\mathrm{AAD}$ group than in aortic tissue from the control group (Figure 5C). Additionally, the elastase level in patients with in-hospital death or without 1-year survival was significantly higher than that in patients without in-hospital death or with 1-year survival (Figures 5D,E).

Local NETosis was detected in the aortic samples from AAD patients, as colocalization of $\mathrm{NE}$ with citH3 was observed by confocal microscopy (Figure 6A). Compared with healthy donor aortic tissue, the numbers of NETs formed per field in the tissue samples from AAD patients were significantly increased (Figure 6B). Moreover, the number of NETs formed per field was positively correlated with the detection risk score of $\mathrm{AD}$ and the APACHE II score (Figures 6C,D). The number of NETs formed per field in patients with in-hospital death or death within 1 -year was significantly higher than that in patients without in-hospital death or death within 1-year (Figures 6E,F). These results indicated that excess NET components in aortic tissue samples are associated with the disease severity and prognosis of AAD. 
A
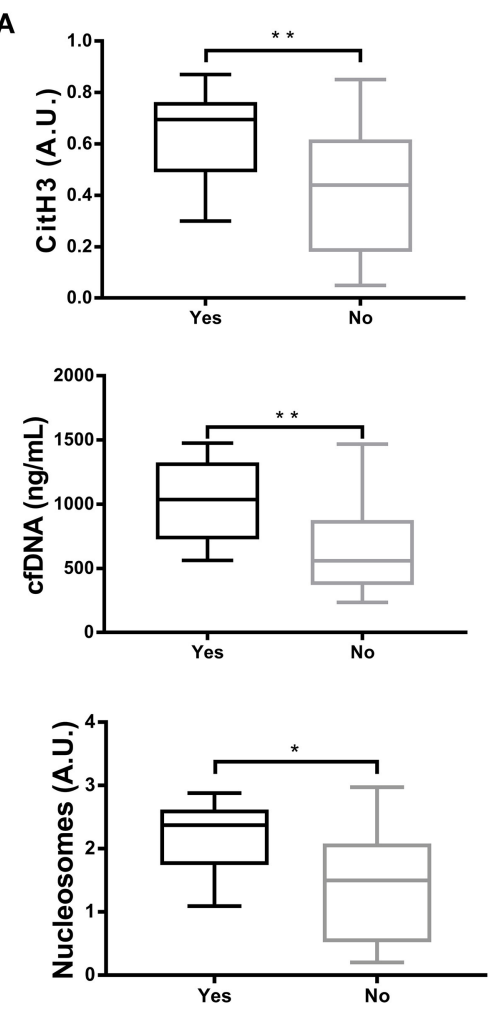

In-hospital death
B
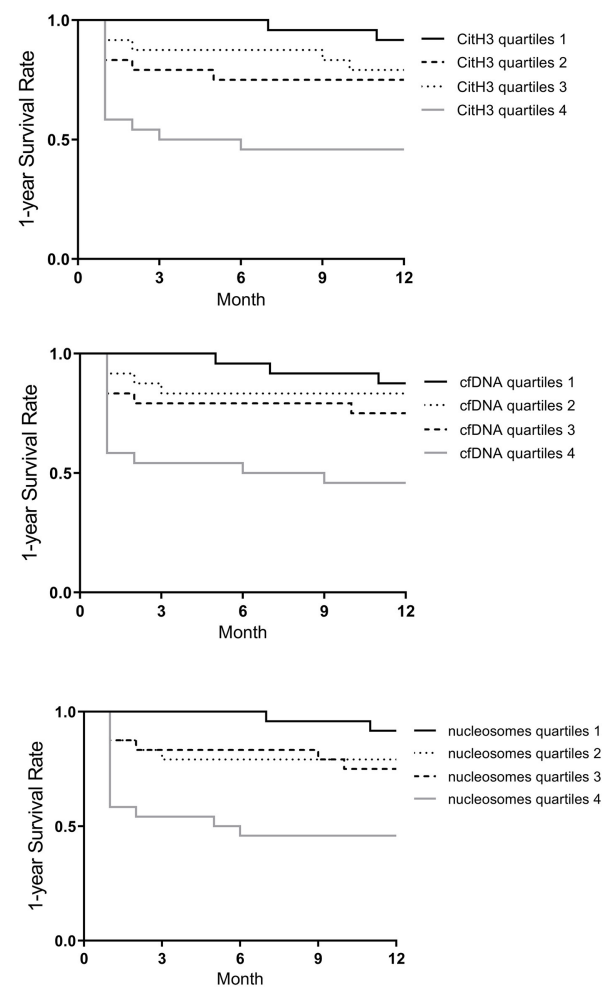

C

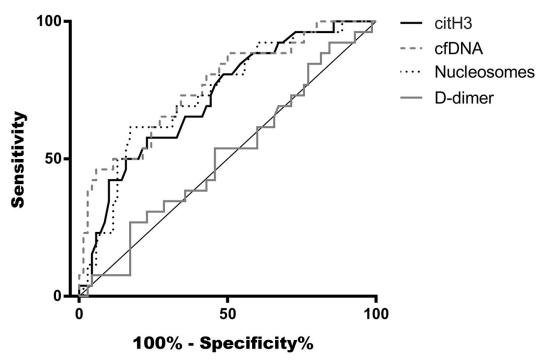

FIGURE 3 | Prognostic significance of circulating NET markers for predicting in-hospital death and 1-year survival of AAD patients. (A) Serum levels of citH3, cfDNA and nucleosomes in patients with in-hospital death were significantly higher than those in patients without in-hospital death. (B) AAD patients with the highest quartiles of citH3, cfDNA or nucleosome levels presented significantly lower survival rates by 1 year than patients with the lower three quartiles of citH3. (C) Based on the ROC curve, three circulating NET markers showed superior prediction ability of 1 -year survival compared with $D$-dimer. ${ }^{\star} P<0.05$, ${ }^{* *} P<0.01$.

\section{DISCUSSION}

To the best of our knowledge, this is the first time that the diagnostic and prognostic value of NETs has been evaluated in patients with AAD. In this study, the major findings were that: (a) circulating NET markers had reliable diagnostic value of AAD, with superior diagnostic performance to discriminate patients with chest pain from other reasons in the emergency department compared with D-dimer; (b) circulating NET markers were independently associated with the disease severity, in-hospital death and 1-year survival of patients with AAD; and (c) excessive NET components in the aortic tissue were associated with the disease severity and prognosis of AAD patients.

It is well-known that neutrophils are the first line of defense against infections by pathogens. Neutrophils are also pivotal as primary effector cells at sites of inflammation, but through regulation of their survival by means of regulated cell death, these cells are also involved in the resolution of inflammation (19). Significant neutrophilic infiltration was found not only in early but also in later stages of organizing dissections and not only in the clot but also in adventitial fat $(10,20)$. This could reflect an upregulation of neutrophil survival to maintain the intense tissue remodeling required for the repair of the arterial wall. In both surgical and autopsy cases of AAD, immunohistochemical staining for citH3 revealed a massive presence of NETs in the clot and in the adventitia in the subacute stage and less abundantly in early organizing stage dissections (10). It is worth noting that aortic tissue samples were collected during surgery in this study. However, in surgical cases, awareness of the pattern of margination, transmigration and extravasation around microvessels that may occur due to robust handling during surgery should be considered to avoid overinterpration of the more diffuse patterns of neutrophil infiltration and NET release related to dissection.

Our results elucidated that the three markers of NETs are significantly elevated in patients with AAD compared with the corresponding levels in subjects from the control group. Recent reports have documented the association of NETs with the severity of stroke evaluated by clinical indexes $(21,22)$. Our study showed for the first time that the three markers of NETs were in line with the disease severity score of AAD at onset and discharge. However, the APAHE II scoring system is predictive of disease severity only when specific baseline and wound characteristics are accounted for. A plasma marker that can be easily and quickly 


\section{A}

\section{Univariate risk factor analysis} 1-year Survival
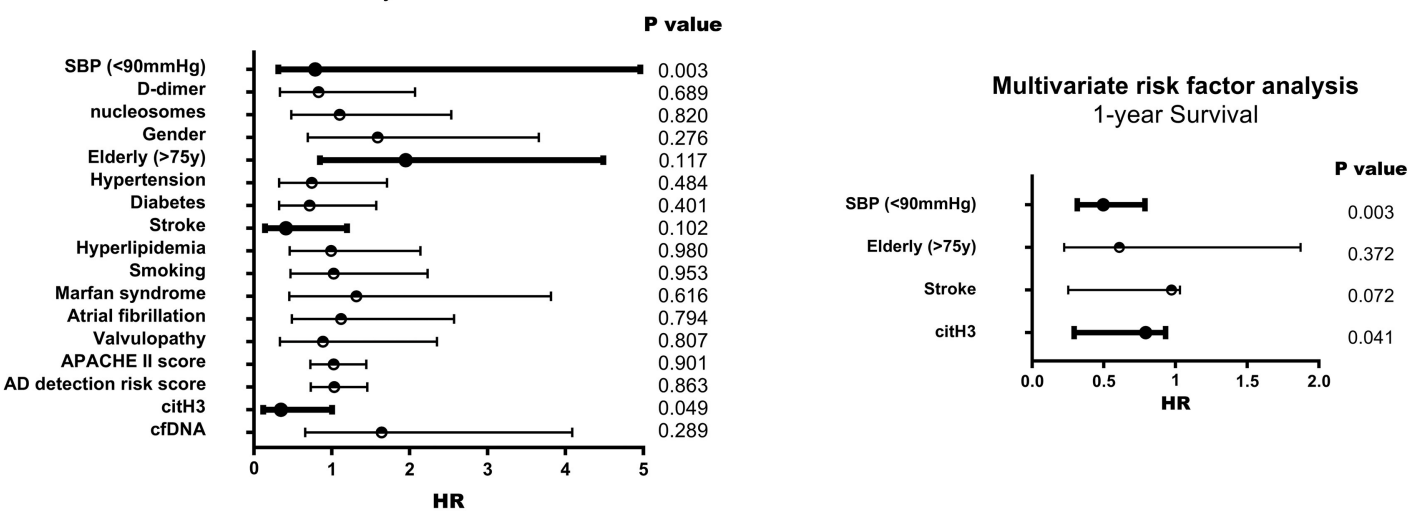

B
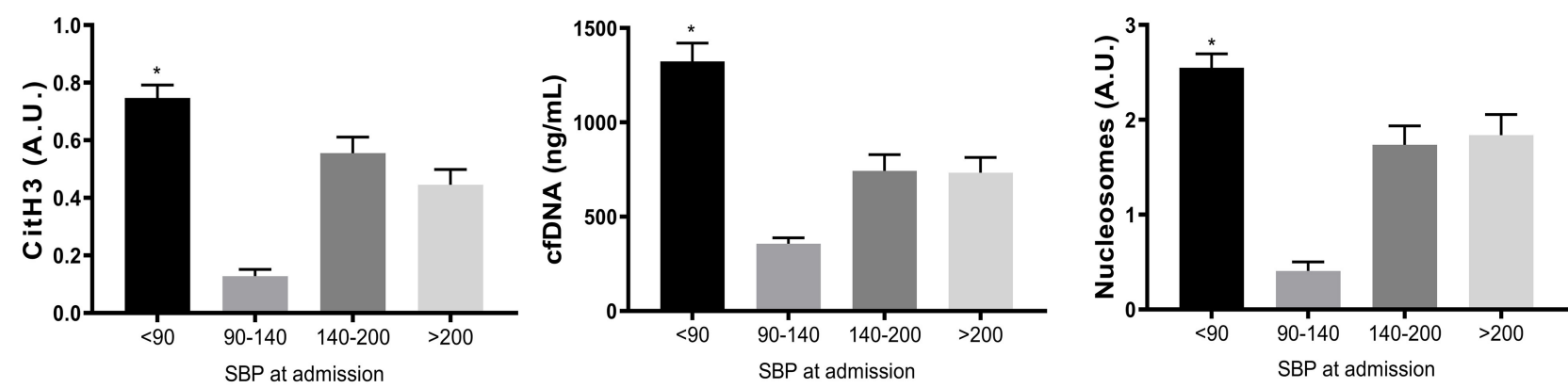

FIGURE 4 | Risk factor analysis of 1-year survival in AAD patients. (A) By univariate and multivariate risk factor analysis, systolic blood pressure (SBP) < 90 mmHg and citH3 levels were identified as independent risk factors for 1-year survival. (B) AAD patients with SBP $<90$ mmHg had significantly increased circulating NET markers compared with patients with SBP $>90 \mathrm{mmHg}$. ${ }^{\star} P<0.05$.

tested to stratify patients with different risks of mortality is of great prognostic significance.

In this study, a time course study of NET markers was performed in AAD patients according to the admission time from symptom onset. The levels of all three NET markers peaked within $12-24 \mathrm{~h}$ after symptom onset. However, the concentrations of NET markers were measured using leftover samples of the patients from initial presentation. However, strictly speaking, the time after the actual onset of disease might be different for each patient. It would be beneficial to study the changes in concentrations according to time course or disease progression intraindividually. In addition, the time window for NET concentrations returning to the baseline level after surgery also needs to be determined. This will provide a stable time horizon for the testing of NET markers to predict the outcome of treatment for patients with AAD.

The mechanism underlying the increased NET formation in AAD remains unknown. AAD is considered an active inflammatory process that occurs in response to endothelial damage through high shear stress. Neutrophils and NETs are emerging as important mediators of pathogenic inflammation in the aorta (23). When aortic dissection occurs, disruption of the aortic media immediately changes aorta hemodynamics, with intramural hemorrhage leading to propagation and tracking of blood within the media, which will overactivate the coagulation system. Currently, there is increasing awareness that NETs are linked to thrombosis since they may shift the hemostatic balance toward excessive coagulation (24). AAD exhibits high concentrations of tissue factor (TF) in serum, and the ability of neutrophils to expose functional TF on NETs is considered a link between inflammation and coagulation $(25,26)$.

In the last two decades, much progress has been made to make effective biochemical diagnoses of $\mathrm{AAD}$, which is an unmet need with lifesaving value. Several promising biomarkers have emerged. Vinculin, lumican, MMP-12 and high levels of ischemia-modified albumin have been considered potential AAD-related serum markers that may assist in the diagnosis and prediction of the in-hospital mortality of patients with AAD (27-30). However, most of these biomarkers are still clinically unavailable. In real-world clinical practice, in patients with acute chest pain and elevated D-dimer, a diagnosis of AAD should be considered. D-dimer might be a useful complementary tool to the current diagnostic work-up of patients with suspected AAD $(31,32)$. D-dimer levels may be useful in risk-stratifying patients with potential $\mathrm{AD}$ to rule out $\mathrm{AD}$ if used within the first $24 \mathrm{~h}$ after symptom onset (33). Nevertheless, D-dimers are not always elevated in patients with AAD (34). The results of this study demonstrated that circulating NET markers showed 

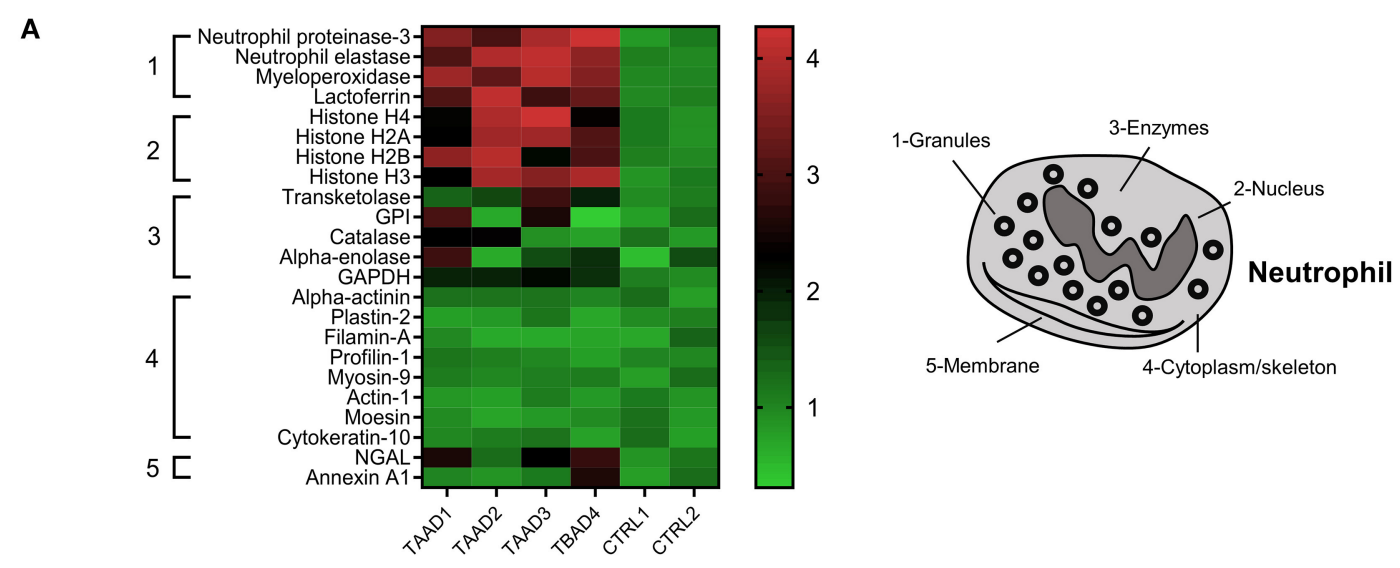

B

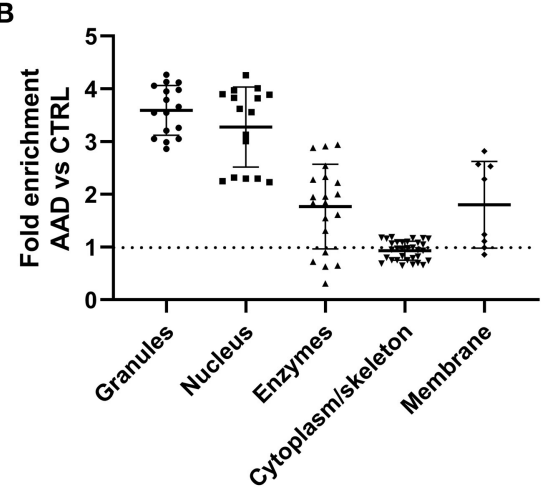

C

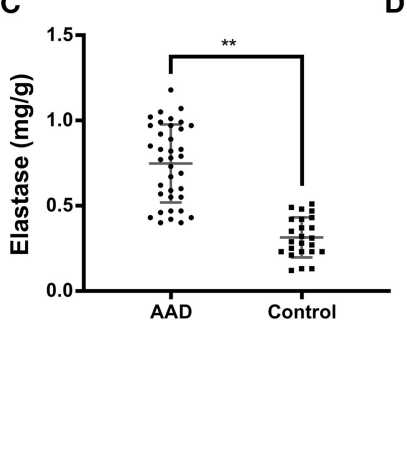

D

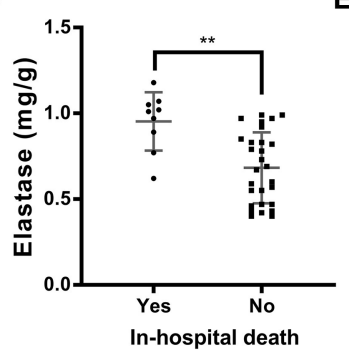

E

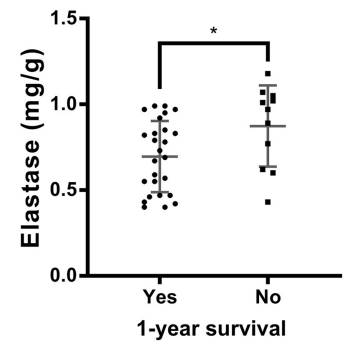

FIGURE 5 | Custom proteomic analysis of aortic tissue lysates from AAD patients. (A) Twenty-three proteins that belonged to five subcellular compartments (nucleus, granules, cytoplasm/cytoskeleton, enzymes, and plasma membrane) were identified to build a custom "NETosis" pathway. Profile plot from the Gene Set Enrichment Analysis (GSEA) showing highly significant enrichment of most proteins in the custom NETosis pathway in the 4 replicates (TAAD1-3, TBAD 4) compared with negative control subjects (CTRL1-2). The false discovery rate-adjusted $\mathrm{P}$ value for this analysis, according to GSEA output, was $<0.01$. (B) The expression of granules and nuclear NET components was significantly higher, and non-granular enzymes and membrane components were mildly enriched in aortic tissue from AAD patients. (C) Neutrophil elastase was significantly higher in aortic tissue from the AAD group than in aortic tissue from the control group. (D,E) The elastase level in patients with in-hospital death or without 1 -year survival was significantly higher than that in patients without in-hospital death or with 1 -year survival. ${ }^{\star} P<0.05$, ${ }^{\star \star} P<0.01$.

significantly superior diagnostic performance compared with Ddimer to discriminate AAD patients with chest pain due to other reasons.

Despite acceptable reproducibility, as indicated by interassay cutoff values, the variability of the data makes clinically relevant cutoffs infeasible, and causality cannot be addressed. Although we used methods in line with current standards, the observed results highlight the need for improved methods when quantifying circulating NET markers (35). However, the inconsistent findings among the levels of cfDNA, citH3 and nucleosomes call into question their specificity in terms of reflecting NETosis. Previous studies have used various methods for evaluating the suitability of NETs as biomarkers in different clinical conditions, in which the main analytical targets were cfDNA, histones, and other components of NETs, such as neutrophil elastase or myeloperoxidase in plasma (36). cfDNA has repeatedly been described as a NET marker due to the objectivity of DNA quantification methods, yet its source is ambiguous, as non-neutrophil cells also release chromatin through cell death processes (35). The citrullination of histone
3 is a necessary step in the formation of NETs, as demonstrated by genetic and pharmacological approaches (37). Thus, citH3 appears to be the most specific marker of NETs, and it has been used to test for the presence of NETs in plasma. Importantly, citH3 has been reported to be independently associated with allcause mortality during the 1-year follow-up in patients with acute ischemic stroke (21). In this study, citH3 was also identified as an independent risk factor for 1-year survival in patients with AAD.

Some limitations should be considered in the interpretation of our results. First, this is a single-center study with a relatively small number of subjects. Potential selection biases are not negligible. Future research on this topic should aim to include a larger study population. Secondly, a long-term follow-up period would be necessary to obtain statistically significant results in the prediction of disease prognoses. In this study, we reported major outcomes observed during the 1-year follow-up. Analysis of the changes in circulating levels of NETs during the follow-up period is also meaningful. Thirdly, our study was mostly descriptive, whereas the specific mechanism of NETs in circulation or aortic tissue promoting the occurrence and progression of AAD was not 


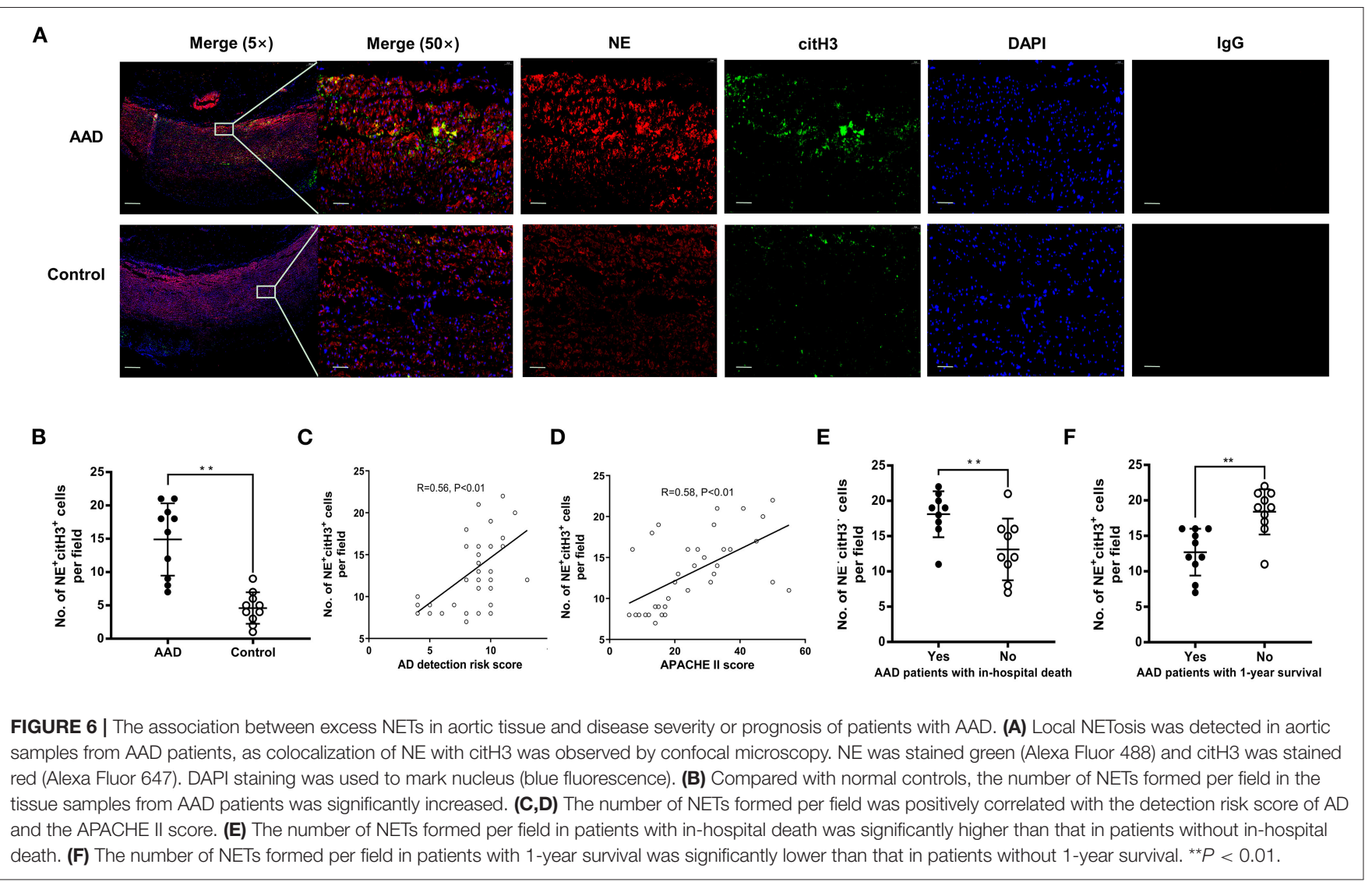

addressed in this study. Further research is needed to determine whether NET components could be used as potential therapeutic targets for AAD.

In conclusion, the present study demonstrates that circulating NET markers have significant diagnostic value for AAD with good diagnostic performance to discriminate patients with chest pain from other causes. NET markers, in both the serum and aortic tissue, are associated with disease severity and the prognosis of AAD patients at the 1-year follow-up. Our results suggest that NETs may constitute a useful diagnostic and prognostic marker in patients with $\mathrm{AAD}$ and open new avenues for future drug therapy for AAD.

\section{DATA AVAILABILITY STATEMENT}

The raw data supporting the conclusions of this article will be made available by the authors, without undue reservation. The data presented in the study are deposited in the (http:// proteomecentral.proteomexchange.org) repository, accession number (PXD001603).

\section{ETHICS STATEMENT}

The studies involving human participants were reviewed and approved by the Ethical Committee of Renji Hospital,
School of Medicine, Shanghai Jiaotong University. The patients/participants provided their written informed consent to participate in this study.

\section{AUTHOR CONTRIBUTIONS}

Study conception and design and drafting of article were performed by SY and YX. Data collection was performed by JC and QN. Analysis and interpretation of data was performed by SY, YD, and XG. Critical revision was performed by GX and XX. All authors contributed to the article and approved the submitted version.

\section{FUNDING}

This work was supported by Grants from the National Science Foundation of China (Grant No. 81700423 and No. 81873526), Clinical Research Innovation and Cultivation Fund of Renji Hospital (Grant No. PYIII17-003), Shanghai Outstanding Young Doctor Training Program from Shanghai Municipal Commission of Health and Family Planning (to SY), Shanghai Jiaotong University Medical Engineering Cross Fund (Grant No. YG 2016QN57), scientific research project of Shanghai municipal commission 
of health and family planning (Grant No. 20164Y0058), Shanghai Jiao Tong University School of Medicine Nursing Research Key Project (Grant No. Jyhz1901), and Hangzhou science and technology development project (Grant No. 20201203B195).

\section{REFERENCES}

1. Bossone E, LaBounty TM, Eagle KA. Acute aortic syndromes: diagnosis and management, an update. Eur Heart J. (2018) 39:739-49d. doi: 10.1093/eurheartj/ehx319

2. Nathan C. Neutrophils and immunity: challenges and opportunities. Nat Rev Immunol. (2006) 6:173-82. doi: 10.1038/nri1785

3. Yoshida S, Yamamoto M, Aoki H, Fukuda H, Akasu K, Takagi K, et al. STAT3 activation correlates with adventitial neutrophil infiltration in human aortic dissection. Ann Vasc Dis. (2019) 12:187-93. doi: 10.3400/avd.oa.19-00007

4. Anzai A, Shimoda M, Endo J, Kohno T, Katsumata Y, Matsuhashi T, et al. Adventitial CXCL1/G-CSF expression in response to acute aortic dissection triggers local neutrophil recruitment and activation leading to aortic rupture. Circ Res. (2015) 116:612-23. doi: 10.1161/CIRCRESAHA.116.304918

5. Kurihara T, Shimizu-Hirota R, Shimoda M, Adachi T, Shimizu $\mathrm{H}$, Weiss SJ, et al. Neutrophil-derived matrix metalloproteinase 9 triggers acute aortic dissection. Circulation. (2012) 126:307080. doi: 10.1161/CIRCULATIONAHA.112.097097

6. Kalkan ME, Kalkan AK, Gündeş A, Yanartaş M, Oztürk S, Gurbuz AS, et al. Neutrophil to lymphocyte ratio: a novel marker for predicting hospital mortality of patients with acute type A aortic dissection. Perfusion. (2017) 32:321-7. doi: 10.1177/0267659115590625

7. Li P, Li M, Lindberg MR, Kennett MJ, Xiong N, Wang Y. PAD4 is essential for antibacterial innate immunity mediated by neutrophil extracellular traps. J Exp Med. (2010) 207:1853-62. doi: 10.1084/jem.20100239

8. Brinkmann V, Reichard U, Goosmann C, Fauler B, Uhlemann Y, Weiss DS, et al. Neutrophil extracellular traps kill bacteria. Science. (2004) 303:15325. doi: 10.1126/science.1092385

9. Döring Y, Libby P, Soehnlein O. Neutrophil extracellular traps participate in cardiovascular diseases: recent experimental and clinical insights. Circ Res. (2020) 126:1228-41. doi: 10.1161/CIRCRESAHA.120.315931

10. Visonà SD, de Boer OJ, Mackaaij C, de Boer HH, Pertiwi KR, de Winter RW, et al. Immunophenotypic analysis of the chronological events of tissue repair in aortic medial dissections. Cardiovasc Pathol. (2018) 34:914. doi: 10.1016/j.carpath.2018.01.009

11. Lim HH, Jeong IH, An GD, Woo KS, Kim KH, Kim JM, et al. Evaluation of neutrophil extracellular traps as the circulating marker for patients with acute coronary syndrome and acute ischemic stroke. J Clin Lab Anal. (2020) 34:e23190. doi: 10.1002/jcla.23190

12. Novotny J, Oberdieck P, Titova A, Pelisek J, Chandraratne S, Nicol P, et al. Thrombus NET content is associated with clinical outcome in stroke and myocardial infarction. Neurology. (2020) 94:e2346-60. doi: 10.1212/WNL.0000000000009532

13. Yang S, Qi H, Kan K, Chen J, Xie H, Guo X, et al. Neutrophil extracellular traps promote hypercoagulability in patients with sepsis. Shock. (2017) 47:1329. doi: 10.1097/SHK.0000000000000741

14. Hiratzka LF, Bakris GL, Beckman JA, Bersin RM, Carr VF, Casey DE Jr, et al. 2010 ACCF/AHA/AATS/ACR/ASA/SCA/SCAI/SIR/STS/SVM Guidelines for the diagnosis and management of patients with thoracic aortic disease. A Report of the American College of Cardiology Foundation/American Heart Association Task Force on Practice Guidelines, American Association for Thoracic Surgery, American College of Radiology, American Stroke Association, Society of Cardiovascular Anesthesiologists, Society for Cardiovascular Angiography and Interventions, Society of Interventional Radiology, Society of Thoracic Surgeons, and Society for Vascular Medicine. J Am Coll Cardiol. (2010) 55:e27-129. doi: 10.1016/j.jacc.2010.02.010

15. Borissoff JI, Joosen IA, Versteylen MO, Brill A, Fuchs TA, Savchenko AS, et al. Elevated levels of circulating DNA and chromatin are independently associated with severe coronary atherosclerosis and a

\section{SUPPLEMENTARY MATERIAL}

The Supplementary Material for this article can be found online at: https://www.frontiersin.org/articles/10.3389/fcvm. 2021.683445/full\#supplementary-material

prothrombotic state. Arterioscler Thromb Vasc Biol. (2013) 33:203240. doi: 10.1161/ATVBAHA.113.301627

16. Fadini GP, Menegazzo L, Rigato M, Scattolini V, Poncina N, Bruttocao A, et al. NETosis delays diabetic wound healing in mice and humans. Diabetes. (2016) 65:1061-71. doi: 10.2337/db15-0863

17. Ravindran M, Khan MA, Palaniyar N. neutrophil extracellular trap formation: physiology, pathology, and pharmacology. Biomolecules. (2019) 9:365. doi: 10.3390/biom9080365

18. Papayannopoulos V. Neutrophil extracellular traps in immunity and disease. Nat Rev Immunol. (2018) 18:134-47. doi: 10.1038/nri.2017.105

19. Witko-Sarsat V, Mocek J, Bouayad D, Tamassia N, Ribeil J-A, Candalh $\mathrm{C}$, et al. Proliferating cell nuclear antigen acts as a cytoplasmic platform controlling human neutrophil survival. J Exp Med. (2010) 207:263145. doi: 10.1084/jem.20092241

20. del Porto F, Proietta M, Tritapepe L, Miraldi F, Koverech A, Cardelli P, et al. Inflammation and immune response in acute aortic dissection. Ann Med. (2010) 42:622-9. doi: 10.3109/07853890.2010.518156

21. Valles J, Lago A, Santos MT, Latorre AM, Tembl JI, Salom JB, et al. Neutrophil extracellular traps are increased in patients with acute ischemic stroke: prognostic significance. Thromb Haemost. (2017) 117:1919-29. doi: 10.1160/TH17-02-0130

22. Tsai NW, Lin TK, Chen SD, Chang WN, Wang HC, Yang TM, et al The value of serial plasma nuclear and mitochondrial DNA levels in patients with acute ischemic stroke. Clin Chim Acta. (2011) 412:4769. doi: 10.1016/j.cca.2010.11.036

23. Liu Y, Carmona-Rivera C, Moore E, Seto NL, Knight JS, Pryor M, et al. Myeloid-specific deletion of peptidylarginine deiminase 4 mitigates atherosclerosis. Front Immunol. (2018) 9:1680. doi: 10.3389/fimmu.2018.01680

24. Gould TJ, Lysov Z, Liaw PC. Extracellular DNA and histones: double-edged swords in immunothrombosis. J Thromb Haemost. (2015) 13(Suppl. 1):S8291. doi: $10.1111 /$ jth.12977

25. Kambas K, Chrysanthopoulou A, Vassilopoulos D, Apostolidou E, Skendros P, Girod A, et al. Tissue factor expression in neutrophil extracellular traps and neutrophil derived microparticles in antineutrophil cytoplasmic antibody associated vasculitis may promote thromboinflammation and the thrombophilic state associated with the disease. Ann Rheum Dis. (2014) 73:1854-63. doi: 10.1136/annrheumdis-2013-203430

26. Gao Z, Pei X, He C, Wang Y, Lu J, Jin M, et al. Oxygenation impairment in patients with acute aortic dissection is associated with disorders of coagulation and fibrinolysis: a prospective observational study. J Thorac Dis. (2019) 11:1190-201. doi: 10.21037/jtd.2019.04.32

27. Gu G, Cheng W, Yao C, Yin J, Tong C, Rao A, et al. Quantitative proteomics analysis by isobaric tags for relative and absolute quantitation identified Lumican as a potential marker for acute aortic dissection. J Biomed Biotechnol. (2011) 2011:920763. doi: 10.1155/2011/920763

28. Proietta M, Tritapepe L, Cifani N, Ferri L, Taurino M, Del Porto F. MMP12 as a new marker of Stanford-A acute aortic dissection. Ann Med. (2014) 46:44-8. doi: 10.3109/07853890.2013.876728

29. Yang G, Zhou Y, He H, Pan X, Chai X. Ischemia-modified albumin, a novel predictive marker of in-hospital mortality in acute aortic dissection patients. Front Physiol. (2019) 10:1253. doi: 10.3389/fphys.2019.01253

30. Wang HQ, Yang $\mathrm{H}$, Tang Q, Gong YC, Fu YH, Wan F, et al. Identification of vinculin as a potential diagnostic biomarker for acute aortic dissection using label-free proteomics. Biomed Res Int. (2020) 2020:7806409. doi: 10.1155/2020/78 06409

31. Ohlmann P, Faure A, Morel O, Petit H, Kabbaj H, Meyer N, et al. Diagnostic and prognostic value of circulating D-Dimers 
in patients with acute aortic dissection. Crit Care Med. (2006) 34:1358-64. doi: 10.1097/01.CCM.0000216686.72457.EC

32. Marill KA. Serum D-dimer is a sensitive test for the detection of acute aortic dissection: a pooled meta-analysis. J Emerg Med. (2008) 34:36776. doi: 10.1016/j.jemermed.2007.06.030

33. Suzuki T, Distante A, Zizza A, Trimarchi S, Villani M, Salerno Uriarte JA, et al. Diagnosis of acute aortic dissection by Ddimer: the International Registry of Acute Aortic Dissection Substudy on Biomarkers (IRAD-Bio) experience. Circulation. (2009) 119:2702-7. doi: 10.1161/CIRCULATIONAHA.108.833004

34. Paparella D, Malvindi PG, Scrascia G, de Ceglia D, Rotunno C, Tunzi F, et al. D-dimers are not always elevated in patients with acute aortic dissection. $J$ Cardiovasc Med. (2009) 10:212-4. doi: 10.2459/JCM.0b013e32831c849e

35. Masuda S, Nakazawa D, Shida H, Miyoshi A, Kusunoki Y, Tomaru U, et al. NETosis markers: quest for specific, objective, and quantitative markers. Clin Chim Acta. (2016) 459:89-93. doi: 10.1016/j.cca.2016. 05.029

36. Jorch SK, Kubes P. An emerging role for neutrophil extracellular traps in noninfectious disease. Nat Med. (2017) 23:279-87. doi: 10.1038/nm.4294

37. Martinod K, Demers M, Fuchs TA, Wong SL, Brill A, Gallant M, et al. Neutrophil histone modification by peptidylarginine deiminase 4 is critical for deep vein thrombosis in mice. Proc Natl Acad Sci USA. (2013) 110:86749. doi: $10.1073 /$ pnas. 1301059110

Conflict of Interest: The authors declare that the research was conducted in the absence of any commercial or financial relationships that could be construed as a potential conflict of interest.

Publisher's Note: All claims expressed in this article are solely those of the authors and do not necessarily represent those of their affiliated organizations, or those of the publisher, the editors and the reviewers. Any product that may be evaluated in this article, or claim that may be made by its manufacturer, is not guaranteed or endorsed by the publisher.

Copyright (C) 2022 Yang, Xiao, Du, Chen, Ni, Guo, Xue and Xie. This is an open-access article distributed under the terms of the Creative Commons Attribution License (CC $B Y)$. The use, distribution or reproduction in other forums is permitted, provided the original author(s) and the copyright owner(s) are credited and that the original publication in this journal is cited, in accordance with accepted academic practice. No use, distribution or reproduction is permitted which does not comply with these terms. 